The University of Southern Mississippi

The Aquila Digital Community

Faculty Publications

$3-1-2000$

\title{
Effect of Interfacial Tension on Propagating Polymerization Fronts
}

R. Texier-Picard

Université Lyon

John A. Pojman

University of Southern Mississippi, john@pojman.com

Vitaly Volpert

Université Lyon

Follow this and additional works at: https://aquila.usm.edu/fac_pubs

Part of the Chemistry Commons

\section{Recommended Citation}

Texier-Picard, R., Pojman, J. A., Volpert, V. (2000). Effect of Interfacial Tension on Propagating Polymerization Fronts. Chaos, 10(1), 224-230.

Available at: https://aquila.usm.edu/fac_pubs/9191

This Article is brought to you for free and open access by The Aquila Digital Community. It has been accepted for inclusion in Faculty Publications by an authorized administrator of The Aquila Digital Community. For more information, please contact Joshua.Cromwell@usm.edu. 


\title{
Effect of interfacial tension on propagating polymerization fronts
}

\author{
R. Texier-Picard \\ Analyse Numérique, Université Lyon 1, UMR 5585 CNRS, 69622 Villeurbanne cedex, France \\ J. A. Pojman \\ Department of Chemistry and Biochemistry, The University of Southern Mississippi, Hattiesburg, \\ Mississippi 39406-5043 \\ Vit. A. Volpert \\ Analyse Numérique, Université Lyon 1, UMR 5585 CNRS, 69622 Villeurbanne cedex, France
}

(Received 5 April 1999; accepted for publication 14 October 1999)

\begin{abstract}
This paper is devoted to the investigation of polymerization fronts converting a liquid monomer into a liquid polymer. We assume that the monomer and the polymer are immiscible and study the influence of the interfacial tension on the front stability. The mathematical model consists of the reaction-diffusion equations coupled with the Navier-Stokes equations through the convection terms. The jump conditions at the interface take into account the interfacial tension. Simple physical arguments show that the same temperature distribution could not lead to Marangoni instability for a nonreacting system. We fulfill a linear stability analysis and show that interaction of the chemical reaction and of the interfacial tension can lead to an instability that has another mechanism: the heat produced by the reaction decreases the interfacial tension and initiates the liquid motion. It brings more monomer to the reaction zone and increases even more the heat production. This feedback mechanism can lead to the instability if the frontal Marangoni number exceeds a critical value. (C) 2000 American Institute of Physics. [S1054-1500(00)01701-8]
\end{abstract}

Similar to gaseous flames, exothermic polymerization fronts can propagate through a medium if the activation energy of the reaction is sufficiently high. If the monomer and polymer are immiscible liquids, the frontal reaction results in a narrow interface moving at constant velocity when the monomer is changed into polymer. The surface tension varies with temperature, which may generate a convective flow in the liquids. We show analytically that under microgravity conditions the interaction of the exothermic chemical reaction and of the surface tension may lead to an instability. We propose experimental conditions to verify these theoretical results.

\section{INTRODUCTION}

Frontal polymerization can be accompanied by various instabilities, such as the thermal instability (see Refs. 1-3, hydrodynamical instabilities (see Refs. 4-7), the RayleighTaylor instability (see Ref. 8). The thermal instability appears because of the competition of heat production due to the chemical reaction and heat diffusion from the reaction zone to the cold reagents. It is well known for combustion (see, for example, Ref. 9) and it leads to periodic oscillations or to various multidimensional modes of the front propagation. The hydrodynamical instability is also observed for the gaseous combustion (see Refs. 10 and 11) and it appears because of the heat expansion of the gas in the reaction zone. The Rayleigh-Taylor instability is caused by the density difference between the reagents and the products of the reaction. It appears as a result of action of the gravity and it can lead to reactive fingering. In this work we study another type of instability of polymerization fronts. We assume that the monomer and the polymer are immiscible liquids, and we study the influence of interfacial tension on the front stability.

To describe expected phenomena, suppose that there is a perturbation at the interface, where the temperature is greater than at other points of the interface. In most cases the interfacial tension decreases with increasing temperature, and we assume that this is true here. Then the liquid will move along the interface from the spot where the perturbation is located. To maintain the continuity of fluid flow the liquid moves to this spot from both sides of the interface. This motion brings to the interface the cold monomer and the hot polymer, which has the same temperature as the interface. Hence the average temperature decreases, and the perturbation decays. Thus the Marangoni instability cannot exist.

On the other hand, we should take into account the chemical reaction. The liquid motion to the interface brings more monomer to the reaction zone. The heat release because of the reaction can increase the temperature perturbation, which in its turn intensifies the liquid motion and leads to the instability.

We show in this work that the instability resulting from the interaction of a chemical reaction and the interfacial tension exists. We find the critical conditions of the instability. The comparison with experimental values of the parameters shows that the conditions for the instability are realistic, but they may be difficult to obtain experimentally. The instability generates a convective flow in the monomer and polymer, which can distort the front.

The experiments must be performed in simulated microgravity conditions to avoid the interference of gravitationally 
induced instabilities. We can choose also the values of parameters when there is no thermal instability. Finally the monomer and the polymer are practically incompressible and this excludes the possibility of the hydrodynamical instability.

\section{MATHEMATICAL MODEL}

We consider a model consisting of equations for the temperature, for the depth of conversion, and of the NavierStokes equations. We assume that

(1) the monomer and the polymer are incompressible, immiscible, have the same density $\rho$, the same coefficient of thermal diffusivity $\kappa$ but different viscosities, $\nu_{1}$ for the polymer and $\nu_{2}$ for the monomer, $\nu_{1} \geqslant \nu_{2}$;

(2) the chemical reaction is a one-step zero-order reaction with the reaction rate given by

$W=k(T) \varphi(\alpha)$,

where $T$ is the temperature, $\alpha$ is the depth of conversion,

$$
\varphi(\alpha)= \begin{cases}1 & \text { if } 0 \leqslant \alpha<1, \\ 0 & \text { if } \alpha=1\end{cases}
$$

and

$$
k(T)=k_{0} \exp (-E /(R T)) .
$$

Here $E$ is the activation energy, $R$ the ideal gas constant, and $k_{0}$ is a pre-exponential factor.

(3) The influence of the walls can be neglected.

(4) The coefficient of mass diffusion is much less than the coefficient of thermal diffusivity, and the mass diffusion can be neglected. This condition holds for frontal polymerization.

Under these assumptions we have the following system of equations:

$$
\begin{aligned}
& \frac{\partial T}{\partial t}+v \cdot \nabla T=\kappa \Delta T+q W \\
& \frac{\partial \alpha}{\partial t}+v \cdot \nabla \alpha=W \\
& \frac{\partial v_{i}}{\partial t}+v \cdot \nabla v_{i}=-\frac{1}{\rho} \frac{\partial p}{\partial x_{i}}+\operatorname{div}\left[\nu\left(\nabla v_{i}+\frac{\partial v}{\partial x_{i}}\right)\right], \quad i=1,2,3
\end{aligned}
$$

$$
\operatorname{div} v=0 .
$$

Here $v=\left(v_{1}, v_{2}, v_{3}\right)$ is the velocity of the medium, $p$ is the pressure, $q$ is the adiabatic heat release, $\nu=\nu(\alpha, T)$ is the viscosity of the medium,

$$
\nabla=\left(\frac{\partial}{\partial x_{1}}, \frac{\partial}{\partial x_{2}}, \frac{\partial}{\partial x_{3}}\right) \quad \text { and } \Delta=\frac{\partial^{2}}{\partial x_{1}^{2}}+\frac{\partial^{2}}{\partial x_{2}^{2}}+\frac{\partial^{2}}{\partial x_{3}^{2}} .
$$

The viscosity dependence on the depth of conversion is essentially stronger than its dependence on temperature. In what follows we consider $\nu$ only as a function of $\alpha$, and we put $\nu(0)=\nu_{2}, \nu(1)=\nu_{1}$.
The system of Eqs. (1)-(4) is considered in the whole space $-\infty<x_{1}, x_{2}, x_{3}<+\infty$. We suppose that the front propagates in the direction $x_{3}$ and that

$$
\begin{aligned}
& T \rightarrow T_{0}, \quad \alpha \rightarrow 0, \quad v \rightarrow 0 \quad \text { as } \quad x_{3} \rightarrow+\infty, \\
& T \rightarrow T_{a}=T_{0}+q, \quad \alpha \rightarrow 1, \quad v \rightarrow 0 \quad \text { as } x_{3} \rightarrow-\infty .
\end{aligned}
$$

Using nondimensional variables and parameters:

$$
\begin{aligned}
& c^{2}=k_{0} \kappa \frac{R T_{a}^{2}}{2 E} \exp \left(-\frac{E}{R T_{a}}\right), \\
& \bar{x}_{i}=\frac{x_{i} c}{\kappa}, \quad i=1,2,3, \quad t_{1}=\frac{t c^{2}}{\kappa}, \quad p_{1}=\frac{c^{2} p}{\rho}, \\
& \theta=\frac{T-T_{a}}{q},
\end{aligned}
$$

and omitting the bar for $x_{i}(\nabla, \Delta$, div, will now be operators related to the new variables) and the subscript 1 , we can rewrite the problem in the form:

$$
\begin{gathered}
\frac{\partial \theta}{\partial t}+v \cdot \nabla \theta=\Delta \theta+Z \exp \left(\frac{\theta}{Z^{-1}+\delta \theta}\right) \varphi(\alpha), \\
\frac{\partial \alpha}{\partial t}+v \cdot \nabla \alpha=Z \exp \left(\frac{\theta}{Z^{-1}+\delta \theta}\right) \varphi(\alpha), \\
\frac{\partial v_{i}}{\partial t}+v \cdot \nabla v_{i}=-\frac{\partial p}{\partial x_{i}}+P \Delta v_{i}+\nabla P \cdot\left(\nabla v_{i}+\frac{\partial v}{\partial x_{i}}\right), \\
i=1,2,3,
\end{gathered}
$$

$\operatorname{div} v=0$

with conditions at infinity:

$$
\begin{aligned}
& \theta \rightarrow 0, \quad \alpha \rightarrow 0, \quad \text { and } v \rightarrow 0 \quad \text { as } \quad x_{3} \rightarrow+\infty, \\
& \theta \rightarrow-1, \quad \alpha \rightarrow 1, \quad \text { and } \quad v \rightarrow 0 \text { as } \quad x_{3} \rightarrow-\infty .
\end{aligned}
$$

Here $Z=q E /\left(R T_{a}^{2}\right)$ is the Zeldovich number, $\delta=R T_{a} / E$, $P=\nu / \kappa, P_{1}=\nu_{1} / \kappa$ is the Prandtl number for the polymer, and $P_{2}=\nu_{2} / \kappa$ is the Prandtl number for the monomer.

It is important to note that a system in which the monomer and its polymer are totally immiscible has not been found yet. However for some polymerization fronts there is a very sharp concentration gradient in the mixing zone, and it is close to an interface separating two liquids. One of them contains mostly the monomer, the other one mostly the polymer (see Fig. 1, or Ref. 7 for other examples).

The goal of this work is to investigate whether the interfacial tension can influence the front stability. In order to study this stability analytically, we employ the so-called infinitely narrow reaction zone method, which assumes that the chemical reaction occurs at a surface $x_{3}=\zeta\left(x_{1}, t\right)$. It is a conventional approach for combustion problems developed by Zeldovich and Frank-Kamenetskii for gaseous combustion (see Ref. 12) and later used also to study condensed phase reaction fronts (see Ref. 13). In our case it means that the monomer is located in the region $x_{3}>\zeta\left(x_{1}, t\right)$, the polymer in $x_{3}<\zeta\left(x_{1}, t\right)$. Then instead of Eqs. (5) and (6) we have outside the reaction surface 


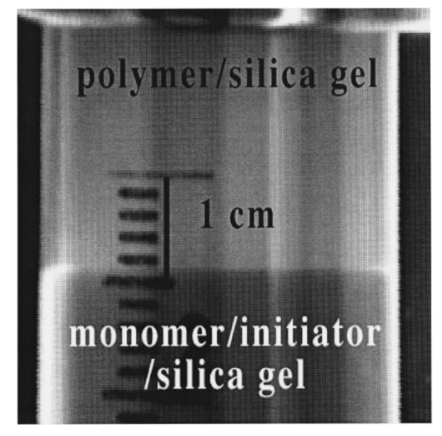

FIG. 1. A polymerization front with a monomer and a polymer separated by a very sharp concentration gradient. Silica gel is used to prevent convection. Adapted from Ref. 2.

$$
\begin{aligned}
& \frac{\partial \theta}{\partial t}+v \cdot \nabla \theta=\Delta \theta, \quad x_{3} \neq \zeta\left(x_{1}, t\right) \\
& \alpha= \begin{cases}0, & x_{3}>\zeta\left(x_{1}, t\right), \\
1, & x_{3}<\zeta\left(x_{1}, t\right) .\end{cases}
\end{aligned}
$$

Equations (7)-(10) should be completed by the jump conditions for the temperature and for the velocity at the interface. We discuss them in the next section.

This problem has a travelling wave solution of the form: $\theta\left(x_{1}, x_{3}, t\right)=\theta_{0}\left(x_{3}-u t\right), \quad \alpha\left(x_{1}, x_{3}, t\right)=\alpha_{0}\left(x_{3}-u t\right), v_{0}=0$. We will analyze its stability. In what follows we assume for simplicity that the temperature, depth of conversion and velocity do not depend on the variable $x_{2}$, and that $v_{2}=0$. It does not change the critical conditions of the instability.

\section{JUMP CONDITIONS AT THE INTERFACE}

\section{A. Conditions for the temperature}

The jump conditions for the temperature have a conventional form (see Refs. 13 and 14) and we recall them briefly.

The temperature is continuous at the interface:

$$
[\theta]_{-}^{+}=0 \text {. }
$$

Here $[\cdots]_{-}^{+}$denotes the jump of a function at the reaction zone, $[f]_{-}^{+}=f(\zeta-0)-f(\zeta+0)$.

We rewrite Eqs. (5) and (6) with the change of variables $z=x_{3}-\zeta\left(x_{1}, t\right)$ so that the reaction front is fixed:

$$
\begin{aligned}
\frac{\partial \theta}{\partial t}= & \frac{\partial^{2} \theta}{\partial z^{2}}+\left(\frac{\partial \zeta}{\partial t}\left(t, x_{1}\right)-v_{z}\right) \frac{\partial \theta}{\partial z} \\
& +Z \exp \left(\frac{\theta}{Z^{-1}+\delta \theta}\right) \varphi(\alpha) \\
\frac{\partial \alpha}{\partial t}= & \left(\frac{\partial \zeta}{\partial t}\left(t, x_{1}\right)-v_{z}\right) \frac{\partial \alpha}{\partial z}+Z \exp \left(\frac{\theta}{Z^{-1}+\delta \theta}\right) \varphi(\alpha) .
\end{aligned}
$$

Subtracting (13) from (12) and integrating with respect to $z$ we find

$$
\left[\theta^{\prime}\right]_{-}^{+}=-\frac{\partial \zeta}{\partial t}+v_{z}\left(0^{+}\right)
$$

Here as well as in the next jump condition we neglect the second order terms. For example, if we put $v=v_{s}+\tilde{v}, \theta$ $=\theta_{s}+\widetilde{\theta}$, where $v_{s}$ and $\theta_{s}$ correspond to the travelling wave solution, and $\tilde{v}$ and $\widetilde{\theta}$ are small perturbations, then the term

$$
v_{1} \frac{\partial \theta}{\partial x_{1}}=\tilde{v} \frac{\partial \widetilde{\theta}}{\partial x_{1}}
$$

is a second order term. It can be neglected in the linear stability analysis.

Multiplying (12) by $\theta^{\prime}$ and integrating with respect to $z$ we find:

$$
\begin{aligned}
{\left[\theta^{\prime 2}\right]_{-}^{+} } & =-2 Z \int_{-1}^{\theta(\zeta)} \exp \left(\frac{\tau}{Z^{-1}+\delta \tau}\right) d \tau \\
& \simeq u^{2}+2 Z \theta(\zeta)
\end{aligned}
$$

Here

$$
u^{2}=-2 Z \int_{-1}^{0} \exp \left(\frac{\tau}{Z^{-1}+\delta \tau}\right) d \tau
$$

is the normal speed of front propagation.

The problem (9), (11), (14), (15) has a travelling wave solution:

$\theta_{s}=\left\{\begin{array}{l}0 \quad \text { if } z=x_{3}-\zeta\left(x_{1}, t\right)<0, \\ -1+\exp (-u z) \quad \text { if } z=x_{3}-\zeta\left(x_{1}, t\right)>0,\end{array} v_{s}=0\right.$.

To fulfill the linear stability analysis we linearize the jump conditions (11), (14), and (15). We put

$$
\begin{aligned}
& \theta=\theta_{s}+\theta_{j}(z) \exp \left(\omega t+i k x_{1}\right), \\
& v=v_{s}+v_{j}(z) \exp \left(\omega t+i k x_{1}\right), \\
& \zeta\left(t, x_{1}\right)=u t+\xi\left(t, x_{1}\right)=u t+\epsilon \exp \left(\omega t+i k x_{1}\right),
\end{aligned}
$$

where $j=1 \quad(j=2)$ corresponds to the region $z<0 \quad(z>0)$. The perturbations $\theta_{j}$ and $v_{j}$ are supposed to be small. Taking into account that

$$
\begin{gathered}
\left.\theta\right|_{\xi \pm 0}=\left.\theta_{s}\right|_{ \pm 0}+\xi \theta_{s}^{\prime}( \pm 0)+\theta_{j}( \pm 0) \exp \left(\omega t+i k x_{1}\right) \\
\left.\frac{\partial \theta}{\partial x_{3}}\right|_{\xi \pm 0}=\left.\theta_{s}^{\prime}\right|_{ \pm 0}+\xi \theta_{s}^{\prime \prime}( \pm 0) \\
+\frac{\partial \theta_{j}}{\partial x_{3}}(\xi \pm 0) \exp \left(\omega t+i k x_{1}\right)
\end{gathered}
$$

and neglecting the second order terms, we obtain

$$
\begin{aligned}
& \theta_{2}(0)-\theta_{1}(0)=\epsilon u, \\
& \theta_{2}^{\prime}(0)-\theta_{1}^{\prime}(0)=-\epsilon\left(\omega+u^{2}\right)+v_{2}(0), \\
& u^{2} \epsilon+\theta_{2}^{\prime}(0)=-\frac{Z}{u} \theta_{1}(0) .
\end{aligned}
$$

\section{B. Conditions for the velocity}

The first two conditions

$$
\left[v_{3}\right]_{-}^{+}=\left[\frac{\partial v_{3}}{\partial x_{3}}\right]_{-}^{+}=0
$$


have a conventional form and come from the assumption that the velocity is continuous at the interface.

The next condition can be obtained from the balance of tangential stresses at the interface (see Ref. 15). In the linear approximation it can be written in the form:

$$
\left[\frac{P}{P_{2}}\left(-\frac{\partial v_{1}}{\partial x_{3}}+\frac{\partial v_{3}}{\partial x_{1}}\right)\right]_{-}^{+}=M\left(\frac{\partial \theta}{\partial x_{1}}+\frac{\partial \theta}{\partial x_{3}} \zeta^{\prime}\right) .
$$

Here $M$ is a nondimensional parameter defined by

$$
M=-\frac{d \sigma}{d T} \frac{q}{c \rho \nu_{2}},
$$

where $\sigma$ is the coefficient of surface tension. It can be called the frontal Marangoni number. If we introduce the width of the preheat zone $h=\kappa / c$ then $M$ takes the form

$$
M=-\frac{d \sigma}{d T} \frac{q h}{\kappa \rho \nu_{2}},
$$

and coincides with the usual Marangoni number.

In the approximation of the infinitely narrow reaction zone the temperature gradient is discontinuous at the interface. Hence the last term in the right-hand side of (20) is not defined. We recall that inside the reaction zone

$$
\frac{\partial \theta}{\partial x_{3}}=-u(\theta+1-\alpha) .
$$

If we assume that the phase transition occurs at a critical depth of conversion $\alpha=\alpha_{c}$ then we can determine the temperature gradient at the interface. Hence we have an additional parameter $\alpha_{c}, 0 \leqslant \alpha_{c} \leqslant 1$. To simplify the computations we put $\alpha_{c}=1$. In this case

$$
\frac{\partial \theta}{\partial x_{3}}=O(\epsilon)
$$

and in the linear stability analysis this term should be omitted.

We differentiate Eq. (20) with respect to $x_{1}$ in order to eliminate the derivatives with respect to $x_{3}$ [using (4)]. The $x_{1}$ derivatives of $P$ are of order $\varepsilon$. Neglecting them, we obtain

$$
\left[\frac{P}{P_{2}}\left(-\frac{\partial^{2} v_{3}}{\partial x_{3}^{2}}+\frac{\partial^{2} v_{3}}{\partial x_{1}^{2}}\right)\right]_{-}^{+}=M \frac{\partial^{2} \theta}{\partial x_{1}^{2}} .
$$

If we assume that $v_{3}=0$ at the interface then (22) has the usual form (see Ref. 16). However, we cannot assume $a$ priori that this condition is satisfied.

To obtain the last jump condition at the interface we consider the case where the interface has a finite width and then we pass to the limit as its width tends to zero. For simplicity we will assume that in the case of the finite width $\alpha$ is continuous in the whole domain and is twice differentiable inside the reaction zone. (For example, $\alpha$ may be a constant on either side of the reaction zone, and linear inside the reaction zone.)
Suppose that $P(\alpha)$ is twice continuously differentiable as a function of $\alpha$. Then $P\left(\alpha\left(x_{3}\right)\right)$ is twice differentiable as a function of $x_{3}$ inside the reaction zone. Up to the high order terms, Eq. (7) becomes

$$
\begin{aligned}
& \frac{\partial v_{1}}{\partial t}=-\frac{\partial p}{\partial x_{1}}+P\left(\frac{\partial^{2} v_{1}}{\partial x_{3}^{2}}-\frac{\partial^{2} v_{3}}{\partial x_{3} \partial x_{1}}\right)+P^{\prime}\left(\frac{\partial v_{1}}{\partial x_{3}}+\frac{\partial v_{3}}{\partial x_{1}}\right), \\
& \frac{\partial v_{3}}{\partial t}=-\frac{\partial p}{\partial x_{3}}+P\left(\frac{\partial^{2} v_{3}}{\partial x_{3}^{2}}+\frac{\partial^{2} v_{3}}{\partial x_{1}^{2}}\right)+2 P^{\prime} \frac{\partial v_{3}}{\partial x_{3}}
\end{aligned}
$$

where $P^{\prime}=\partial P / \partial x_{3}$. Applying twice the operator rotation in order to eliminate the pressure gradient and the terms in $v_{1}$ we find

$$
\begin{aligned}
-\frac{\partial}{\partial t} \Delta v_{3}= & P\left(-\frac{\partial^{4} v_{3}}{\partial x_{3}^{4}}-2 \frac{\partial^{4} v_{3}}{\partial x_{3}^{2} \partial x_{1}^{2}}-\frac{\partial^{4} v_{3}}{\partial x_{1}^{4}}\right) \\
& +2 P^{\prime}\left(-\frac{\partial^{3} v_{3}}{\partial x_{3}^{3}}-\frac{\partial^{3} v_{3}}{\partial x_{1}^{2} \partial x_{3}}\right) \\
& +P^{\prime \prime}\left(-\frac{\partial^{2} v_{3}}{\partial x_{3}^{2}}+\frac{\partial^{2} v_{3}}{\partial x_{1}^{2}}\right)
\end{aligned}
$$

Looking for $v_{3}$ in the form $v_{3}\left(x_{1}, x_{3}, t\right)=v\left(x_{3}\right.$ $-u t) \exp \left(\omega t+i k x_{1}\right)$, we obtain

$$
\begin{aligned}
& \left(P v^{\prime \prime}\right)^{\prime \prime}+k^{2}\left(P^{\prime} v-3 P v^{\prime}\right)^{\prime}+u v^{\prime \prime \prime}+\left(P k^{2}-\omega\right) v^{\prime \prime} \\
& -k^{2} u v^{\prime}+k^{2}\left(P k^{2}+\omega\right) v=0 .
\end{aligned}
$$

Suppose that $P\left(\alpha\left(x_{3}\right)\right)$ is continuously differentiable in the whole domain. For example, $\alpha$ is continuously differentiable, or $\alpha$ only satisfies a Lipschitz condition at the boundary of the reaction zone, and the derivative of $P(\alpha)$ satisfies $\left.P^{\prime}(\alpha)\right|_{\alpha=0}=\left.P^{\prime}(\alpha)\right|_{\alpha=1}=0$. We integrate the equality over the reaction zone. Taking into account that $v^{\prime \prime}$ is bounded, $P^{\prime}$ is continuous and equals 0 outside of the transition zone we obtain in the limit as its width tends to zero,

$$
\left[P v^{\prime \prime \prime}-3 k^{2} P v^{\prime}+u v^{\prime \prime}\right]_{-}^{+}=0 .
$$

For an unmovable interface $(u=0)$ this jump condition coincides with that in Ref. 16 . We note that without the assumption that the function $P$ is twice differentiable there are some additional jump conditions for the velocity (cf. Ref. 17 ). However it does not mean that the problem is overdetermined. Indeed the problem (23) is not equivalent to the problem (24), and we need additional conditions to determine arbitrary functions appearing in (24).

\section{LINEAR STABILITY ANALYSIS}

We obtain the following linearized problems: $z<0$ (polymer),

$$
\begin{aligned}
& \theta_{1}^{\prime \prime}+u \theta_{1}^{\prime}+\left(\omega-k^{2}\right) \theta_{1}=0 \\
& \omega\left(v_{1}^{\prime \prime}-k^{2} v_{1}\right)=P_{1}\left(v_{1}^{\prime \prime \prime \prime}-2 k^{2} v_{1}^{\prime \prime}+k^{4} v_{1}\right)+u\left(v_{1}^{\prime \prime \prime}-k^{2} v_{1}^{\prime}\right)
\end{aligned}
$$

$z>0$ (monomer)

$\theta_{2}^{\prime \prime}+u \theta_{2}^{\prime}+\left(\omega-k^{2}\right) \theta_{2}=-u v_{2} e^{-u z_{2}}$, 


$$
\begin{aligned}
& \omega\left(v_{2}^{\prime \prime}-k^{2} v_{2}\right)=P_{2}\left(v_{2}^{\prime \prime \prime \prime}-2 k^{2} v_{2}^{\prime \prime}+k^{4} v_{2}\right)+u\left(v_{2}^{\prime \prime \prime}-k^{2} v_{2}^{\prime}\right) \\
& \quad z=0 \text { (interface) } \\
& \theta_{2}(0)-\theta_{1}(0)=\epsilon u \\
& \theta_{2}^{\prime}(0)-\theta_{1}^{\prime}(0)=-\epsilon\left(\omega+u^{2}\right)+v_{1}(0) \\
& u^{2} \epsilon+\theta_{2}^{\prime}(0)=-\frac{Z}{u} \theta_{1}(0) \\
& v_{1}(0)=v_{2}(0), \\
& v_{1}^{\prime}(0)=v_{2}^{\prime}(0), \\
& P_{2}\left(v_{2}^{\prime \prime}(0)+k^{2} v_{2}(0)\right)=P_{1}\left(v_{1}^{\prime \prime}(0)+k^{2} v_{1}(0)\right) \\
& \quad+M P_{2} k^{2} \theta_{1}(0) \\
& P_{2} v_{2}^{\prime \prime \prime}(0)-3 k^{2} P_{2} v_{2}^{\prime}(0)+u v_{2}^{\prime \prime}(0) \\
& \quad=P_{1} v_{1}^{\prime \prime \prime}(0)-3 k^{2} P_{1} v_{1}^{\prime}(0)+u v_{1}^{\prime \prime}(0)
\end{aligned}
$$

We recall that $\theta_{1}, \theta_{2}, v_{1}, v_{2}$ are bounded at $\pm \infty$.

The condition of nontrivial solvability of the system (26) -(36) gives the stability boundary. We want to find the cellular stability boundary, i.e., the case when the eigenvalue with maximum real part is 0 . The system (26)-(29) can be solved explicitly:

$$
\begin{aligned}
& v_{1}(z)=c_{1} e^{k z}+c_{2} e^{\lambda_{2} z}, z<0, \\
& \theta_{1}(z)=c_{3} e^{\mu_{1} z}, z<0, \\
& v_{2}(z)=c_{4} e^{-k z}+c_{5} e^{\lambda_{4} z}, z>0, \\
& \theta_{2}(z)=c_{6} e^{\mu_{2} z}+c_{7} e^{-(k+u) z}+c_{8} e^{\left(\lambda_{4}-u\right) z}, z>0 .
\end{aligned}
$$

Here

$$
\begin{aligned}
& \lambda_{2}=\frac{-u}{2 P_{1}}+\sqrt{\frac{u^{2}}{4 P_{1}^{2}}+k^{2}}, \quad \lambda_{4}=\frac{-u}{2 P_{2}}-\sqrt{\frac{u^{2}}{4 P_{2}^{2}}+k^{2}}, \\
& \mu_{1}=\frac{-u}{2}+\sqrt{\frac{u^{2}}{4}+k^{2}}, \quad \mu_{2}=\frac{-u}{2}-\sqrt{\frac{u^{2}}{4}+k^{2}} .
\end{aligned}
$$

Substituting solutions (37)-(40) into (31)-(36), we obtain a system of linear algebraic equations. The condition of its nontrivial solvability can be written in the form

$$
M=\left(\sqrt{u^{2}+4 k^{4}}-u+\frac{2 Z}{u}\right) \frac{H}{k P_{2}},
$$

where

$$
\begin{aligned}
H= & \frac{\left(P_{1}-P_{2}\right)\left[2 k^{2} u+k\left(\lambda_{4}-\lambda_{2}\right) u+\left(\lambda_{4}+k\right)\left(\lambda_{2}-k\right)\left(P_{1}\left(k-\lambda_{2}\right)-P_{2}\left(k+\lambda_{4}\right)\right)\right]}{2\left(P_{1}-P_{2}\right)\left(\lambda_{4}+k\right)\left(\lambda_{2}-k\right)+u\left(\lambda_{4}-\lambda_{2}+2 k\right)} \\
& +\frac{u^{2}\left(\lambda_{4}-\lambda_{2}\right)}{2\left(P_{1}-P_{2}\right)\left(\lambda_{4}+k\right)\left(\lambda_{2}-k\right)+u\left(\lambda_{4}-\lambda_{2}+2 k\right)} .
\end{aligned}
$$

We denote $M_{\mathrm{cr}}$ the right-hand side in (41). If $M$ is greater than the critical value given by (41) then the instability may occur, whereas if $M<M_{\text {cr }}$ the stationary solution will be stable. We study the dependence of $M_{\mathrm{cr}}$ on parameters in two limiting cases: $P_{1}=P_{2}$, and $P_{1} \rightarrow+\infty$.

If $P_{1}=P_{2}=P$, then

$$
\lambda_{2}=\frac{-u}{2 P}+\sqrt{\frac{u^{2}}{4 P^{2}}+k^{2}}, \quad \lambda_{4}=\frac{-u}{2 P}-\sqrt{\frac{u^{2}}{4 P^{2}}+k^{2}},
$$

and $H$ has a relatively simple form:

$$
H=\frac{u \sqrt{u^{2} / P^{2}+4 k^{2}}}{\sqrt{\left(u^{2} / P^{2}\right)+4 k^{2}}-2 k} .
$$

We note that this expression and consequently $M_{\text {cr }}$ are positive.

$$
\begin{aligned}
& \text { If } P_{1} \rightarrow+\infty \text {, then } \\
& \begin{aligned}
P_{1}\left(k-\lambda_{2}\right) & =P_{1} k+\frac{u}{2}-\sqrt{\frac{u^{2}}{4}+P_{1}^{2} k^{2}} \\
& =P_{1} k+\frac{u}{2}-P_{1} k\left(1+\frac{u^{2}}{8 P_{1}^{2} k^{2}}\right)+o\left(\frac{1}{P_{1}}\right) \\
& =\frac{u}{2}-\frac{u^{2}}{8 P_{1} k}+o\left(\frac{1}{P_{1}}\right)
\end{aligned}
\end{aligned}
$$

and $H=\mathcal{N} / \mathcal{D}$ where

$$
\begin{aligned}
\mathcal{N}= & P_{1} k u\left(k+\lambda_{4}\right)+u^{2}\left(\lambda_{4}-k\right)+o\left(P_{1}\right) \\
\sim & P_{1} k\left(k+\lambda_{4}\right) u<0, \\
\mathcal{D}= & \left(\lambda_{4}+k\right)\left(2 P_{1}\left(\lambda_{2}-k\right)+u\right) \\
& +\left(2 P_{2}\left(\lambda_{4}+k\right)+u\right)\left(k-\lambda_{2}\right) \\
= & \left(\lambda_{4}+k\right) \frac{u^{2}}{4 P_{1} k}+\frac{u}{2 P_{1}}\left(2 P_{2}\left(\lambda_{4}+k\right)+u\right)+o\left(\frac{1}{P_{1}}\right) .
\end{aligned}
$$

But $\lambda_{4}+k<0$ and $2 P_{2}\left(\lambda_{4}+k\right)+u<0$ so $\mathcal{D}<0$. Hence $H$ $\rightarrow+\infty$ as $P_{1} \rightarrow+\infty$. More precisely, $H \sim \alpha P_{1}^{2}$.

Another interesting issue is the behavior of $M_{\text {cr }}$ as $k$ $\rightarrow 0, P_{1}$ and $P_{2}$ being kept constant. It is easy to observe that in this case, $\lambda_{2} \rightarrow 0, \lambda_{4} \rightarrow-u / P_{2}$ so that $H \rightarrow u \neq 0$. It shows that $M_{\mathrm{cr}}$ tends to infinity as $k \rightarrow 0$. Consequently $M_{\mathrm{cr}}(k)$ reaches a minimal value for a certain $k=k^{*}$.

In the general case the stability boundary cannot be found analytically, but a numerical study shows that $M_{\mathrm{cr}}$ is always positive (see Fig. 2), which is physically consistent, and proves that the loss of stability may occur. It is easy to compute the stream function, and to observe what the instability would look like (see Fig. 3). The zero of the vertical coordinate represents the interface, and we can observe a strong vortex above it (in the monomer) caused by this in- 


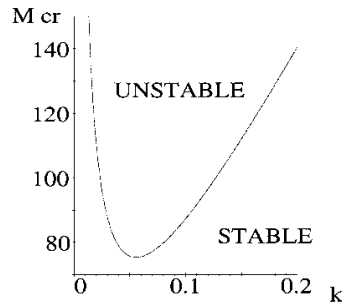

(a)

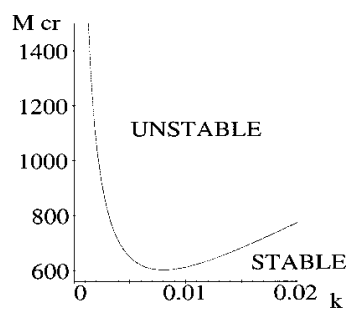

(b)

FIG. 2. $M_{\text {cr }}$ as a function of the wave number $k$ for $\nu_{2}=0.01 \mathrm{~cm}^{2} / \mathrm{s}$ and (a) $\nu_{1}=\nu_{2}$, (b) $\nu_{1}=10 \nu_{2}$.

stability. We can see that the streamlines intersect the interface, because of the chemical reaction (the monomer is changed into polymer). We have to point out that Fig. 3 represents the velocity field in coordinates attached to the front. We also observe in the polymer a counter-vortex induced by the vortex in the monomer. In Fig. 3(b) the polymer is more viscous, therefore the streamlines cannot cross the interface so easily and are flat near the interface.

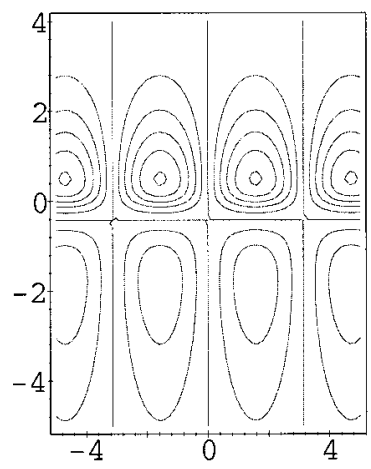

(a)

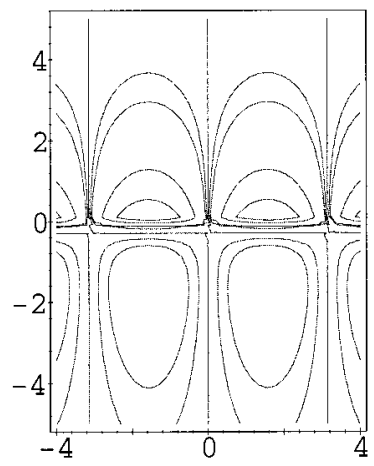

(b)

FIG. 3. Streamlines of the velocity perturbation (a) $\nu_{1}=\nu_{2}$, (b) $\nu_{1}=2 \nu_{2}$.
TABLE I. Comparison of experimental and critical Marangoni numbers for various viscosities: in cases 1, 2, and 3, instability may occur, and the corresponding spatial period is indicated.

\begin{tabular}{cccccc}
\hline \hline Case & $\nu_{2}\left(\mathrm{~cm}^{2} / \mathrm{min}\right)$ & $\nu_{1}\left(\mathrm{~cm}^{2} / \min \right)$ & $M_{\exp }$ & $\min$ of $M_{\mathrm{cr}}$ & period $(\mathrm{cm})$ \\
\hline 1 & 1.5 & 1.5 & 840 & 75 & 28.5 \\
2 & 1.5 & 15 & 840 & 600 & 190.4 \\
3 & 6 & 6 & 210 & 75 & 78.5 \\
4 & 6 & 60 & 210 & 610 & \\
\hline \hline
\end{tabular}

\section{COMPARISON WITH THE EXPERIMENTAL VALUES OF PARAMETERS}

We take the values of parameters corresponding to the polymerization of benzyl acrylate in DMF (see Ref. 7).

- Front velocity: $c=0.6 \mathrm{~cm} / \mathrm{min}$,

- Adiabatic heat release: $q=140 \mathrm{~K}$,

- Thermal conductivity: $\kappa=6 \times 10^{-2} \mathrm{~cm}^{2} / \mathrm{min}$,

- Density: $\rho=1 \mathrm{~g} / \mathrm{cm}^{3}$.

The problem for this comparison is to find an estimate for the derivative of surface tension coefficient with respect to temperature. Indeed we know of no experimental system in which the monomer is immiscible with its molten polymer. Further investigation is necessary in this domain. Even for partially miscible systems we know the values of surface tension, but not of their derivatives with respect to temperature. Our estimate is based on the conjecture that the proportion between the coefficients of surface tension for the air/ water interface and the monomer/polymer interface can be the same as the proportion between their derivatives. Consequently we put $d \sigma / d T=-5.4 \mathrm{~g} /\left(\mathrm{min}^{2} \mathrm{~K}\right)=-1.5 \mathrm{mN} /(\mathrm{m} \mathrm{K})$.

We vary the parameters $\nu_{1}$ and $\nu_{2}$ and find the experimental value $M_{\text {exp }}$ by the formula (21), the minimum of the function $M_{\text {cr }}$ given by (41) and the corresponding value of the wave number $k^{*}$ (see Table I). The wave number determines the spatial period $d$ of the convective structure, $d$ $=2 \pi / k$.

We see that in the cases $1-3, M_{\exp }$ is greater than the minimum of $M_{\mathrm{cr}}$, and the instability may occur. However the period is too large to observe the instability experimentally because it is quite greater than the realistic diameters of experimental tubes. Let us rather consider the wave numbers corresponding to the diameters of the tubes $d_{a}=1.5 \mathrm{~cm}$ and $d_{b}=3 \mathrm{~cm}$, and compute the corresponding values of the critical Marangoni number. The wave numbers corresponding to the diameters $d_{a}$ and $d_{b}$ are $k_{a} \simeq 0.42$ and $k_{b} \simeq 0.21$, respectively. The values of the critical Marangoni number for $k$ $=k_{a}$ (respectively, $k=k_{b}$ ) equal 690 (respectively, 340) in case 1, 21000 (respectively, 10 500) in case 2, and 2800 (respectively, 1420) in case 3. Hence for $k=k_{a}$ as well as for $k=k_{b}$ the instability can be observed only in the first case.

If we fix $\nu_{2}=1.5 \mathrm{~cm}^{2} / \mathrm{min}$, and $k=k_{b}$, then the instability can be observed for $1.5 \mathrm{~cm}^{2} / \mathrm{min} \leqslant \nu_{1} \leqslant 3.18 \mathrm{~cm}^{2} / \mathrm{min}$. (The maximum viscosity for the polymer is only a little more than twice the viscosity of the monomer.) For $k=k_{a}$, the condition is: $1.5 \mathrm{~cm}^{2} / \mathrm{min} \leqslant \nu_{1} \leqslant 1.8 \mathrm{~cm}^{2} / \mathrm{min}$. We see that the viscosity of the product of the reaction should be sufficiently close to the viscosity of the monomer in order to observe the instability. Hence the molecular weight of the 
polymer chains should be very low, which can be achieved experimentally by the addition of a chain transfer agent (Ref. $4)$.

\section{CONCLUSIONS AND DISCUSSION}

In this work we study theoretically exothermic reaction fronts propagating in liquids. We find analytically that a new type of instability may occur as a result of interaction of chemistry and hydrodynamics. It can be explained as follows: suppose that there is a small perturbation of the stationary state, and that the temperature increases locally at the front. This can lead to a weak convective motion that brings fresh monomer to the reaction zone. Though this fresh monomer is colder than the front, it speeds up the reaction and raises the heat release, so that the perturbation amplifies, and the process may lead to an instability.

We find the critical condition when this instability appears: it has the form $M>M_{\mathrm{cr}}\left(P_{1}, P_{2}, u, k\right)$, i.e., the frontal Marangoni number $M$ should be greater than a critical value depending on the Prandtl numbers $P_{1}$ (polymer) and $P_{2}$ (monomer), on the front velocity $u$, and on the wave number of the perturbation $k$. We have shown that this critical value has a minimum as a function of $k$.

The critical conditions of the instability correspond to physically realistic values of parameters though it may be difficult to find an appropriate experimental system. Indeed in most cases the instability cannot be observed experimentally because the wave number $k$ of the perturbation is too small (see Sec. V). If we match the value of $k$ with the dimensions of the tube, we obtain that instability can be observed for example in the case where $\nu_{1}=3 \mathrm{~cm}^{2} / \mathrm{min}, \nu_{2}$ $=1.5 \mathrm{~cm}^{2} / \mathrm{min}$. In order to verify these results experimentally we should reduce the viscosity of the product of the reaction as much as possible. This is experimentally possible, but there is a risk that if the polymer is only a little more viscous than the monomer then they may be miscible, which does not agree with our model.

We have considered the case where monomer and polymer have the same density, but this model does not totally agree with the reality. Physically the polymer is always more dense than the monomer.

In Sec. V we assumed that the derivative of the coefficient of surface tension with respect to the temperature was one percent of the value for the water/air interface. Actually it is difficult to estimate $\sigma$ for a monomer/polymer interface, because experimentally a completely immiscible system is not yet found. Therefore this value was estimated for a miscible system. It can also be estimated for immiscible polymer melts. See, for example, Ref. 18 for polystyrene and polymethyl methacrylate (Ref. 19), for low molecular weight polypropylene glycol and polyethylene glycol, and Ref. 20 for the polypropylene/polystyrene interface.

The values we used for benzyl acrylate in DMF correspond to a solution polymerization. For a solvent-free system, the heat release $q$ is much larger, and we may have $q$ $=200 \mathrm{~K}$. Thus this would aid observing the instability.

We should note finally that this study cannot be experimentally realized under usual gravity $(1 \mathrm{~g})$ because gravity leads to convection of higher amplitude than the weak convective motion due to the perturbation of surface tension. It is well-known that for descending front there is the Rayleigh-Taylor instability and for ascending, simple convection (see Refs. 2 and 7). Thus, a microgravity investigation is required.

${ }^{1}$ V. M. Ilyashenko and J. A. Pojman, Chaos 8, 285 (1998).

${ }^{2}$ J. A. Pojman, V. M. Ilyashenko, and A. M. Khan, J. Chem. Soc., Faraday Trans. 92, 2825-2837 (1996).

${ }^{3}$ Vit. A. Volpert, Vl. A. Volpert, S. P. Davtyan, I. N. Megrabova, and N. F. Surkov, SIAM J. Appl. Math. 52, 368-383 (1992).

${ }^{4}$ M. Garbey, A. Taïk, and V. Volpert, Q. Appl. Math. 56, 1-35 (1998).

${ }^{5}$ Vit. A. Volpert, Vl. A. Volpert, J. A. Pojman, and S. E. Solovyov, Euro. J. Appl. Math. 7, 303-320 (1996).

${ }^{6}$ G. Bowden et al., J. Phys. Chem. B 101, 678 (1997).

${ }^{7}$ B. McCaughey, J. A. Pojman, C. Simmons, and V. A. Volpert, Chaos 8, 520 (1998).

${ }^{8}$ J. A. Pojman, A. M. Khan, and L. J. Mathias, Microgravity Sci. Technol. 10, 36 (1997).

${ }^{9}$ B. J. Matkowsky and G. I. Sivashinsky, SIAM (Soc. Ind. Appl. Math.) J. Appl. Math. 35, 465-478 (1978).

${ }^{10}$ L. D. Landau and E. M. Lifschitz, Fluid Mechanics (Pergamon, Oxford, 1987).

${ }^{11}$ Ya. B. Zeldovich, G. I. Barenblatt, V. B. Librovich, and G. M. Makhviladze, The Mathematical Theory of Combustion and Explosion (Consultants Bureau, New York, 1985).

${ }^{12}$ Ya. B. Zeldovich and D. A. Frank-Kamenetsky, Acta Physicochim. URSS 9, 341-350 (1938).

${ }^{13}$ B. V. Novozhilov, Dokl. Phys. Chem. 141, 836-838 (1961).

${ }^{14}$ M. Garbey, A. Taik, and V. Volpert, Q. Appl. Math. 54, 225-247 (1996).

${ }^{15}$ S. H. Davis and G. M. Homsy, J. Fluid Mech. 98, 527-553 (1980).

${ }^{16}$ A. X. Zhao, C. Wagner, R. Narayanan, and R. Friedrich, Proc. R. Soc. London, Ser. A 451, 487-502 (1995).

${ }^{17}$ E. N. Ferm and D. J. Wolkind, J. Non-Equilib. Thermodyn. 7, 169-190 (1981).

${ }^{18}$ H. Gramespacher and J. Meissner, J. Rheol. 36, 1127-1141 (1992).

${ }^{19}$ F. Quirion and J. Pageau, J. Polym. Sci., Part B: Polym. Phys. 33, 18671875 (1995).

${ }^{20}$ N. R. Demarquette and M. R. Kamal, Polym. Eng. Sci. 34, 1823-1833 (1994). 
Chaos is copyrighted by AIP Publishing LLC (AIP). Reuse of AIP content is subject to the terms at: http://scitation.aip.org/termsconditions. For more information, see http://publishing.aip.org/authors/rights-and-permissions. 